\title{
Clinical usefulness and challenges of instrumented motion analysis in patients with intellectual disabilities
}

\author{
NARRATIVE REVIEW
}

\section{ABSTRACT}

Background: Clinical laboratory testing of locomotor disorders is challenging in patients with intellectual disability (ID). Nevertheless, also in this population gait analysis has substantial value as motor problems are common. To promote its use, adequate protocols need to be developed and the impact on clinical decision making needs to be documented.

Research Question: What is the clinical usefulness of instrumented motion analysis in patients with ID?

Method: This narrative review consists of three parts. A literature review was performed to describe the gait pattern of patients with ID. Next, benefits and challenges of standard gait analysis protocols are described. Finally, a case of a girl with ID due to genetic cause showing gait abnormalities is discussed.

Results: The literature review resulted in 20 studies on "gait" in patients with an "ID", published since August, $1^{\text {st }}$ 2013. Gait deviations were observed in all studies investigating the ID population with an underlying genetic syndrome. Observed gait deviations in the ID population might be attributed to physical characteristics, cognitive components or both. The main goal of clinical gait assessment is the identification of gait deviations and the evaluation of their progress over time, in order to optimize the treatment plan. The choice of adequate method and measurement modalities depends on the clinical goal, the available resources and the abilities of the patient. In the case report we presented, we succeeded in performing an instrumented 3D gait analysis in a girl with severe ID at the ages of 4y4m, $6 \mathrm{y} 0 \mathrm{~m}, 7 \mathrm{y} 2 \mathrm{~m}$ and $8 \mathrm{y} 2 \mathrm{~m}$. Progressive gait deviations were found suggesting a crouch gait pattern was developing. Results of the gait analysis led to the prescription of rigid ankle-foot orthoses.

Significance: Gait analysis has substantial value for patients with ID. Gait analysis allows clinicians to objectify the relationship between physical characteristics and gait features. 


\section{INTRODUCTION}

Gait analysis, when combined with physical examination, provides quantitative information to guide treatment of gait disorders and assess its outcome. Since the 1990s gait analysis has become standard procedure in the treatment of gait problems in children with cerebral palsy [1]. Gait analysis affords the confidence not provided by clinical examination that the correct number and selection of treatment procedures can be chosen $[2,3]$. Despite the value of gait analysis, clinical laboratory testing of locomotor disorders is not yet wide spread in other populations than children with cerebral palsy or post-stroke patients. The issue of use does not relate to its perceived value but to the challenges faced by the gait analysis protocol [4]. Especially in patients with intellectual disability (ID), difficulties in understanding instructions and lack of body awareness during physical examination could negatively affect the result of the gait analysis.

Nevertheless, gait analysis has substantial value for patients with ID. Gait is a highly relevant functional motor skill. In the older adult population, poor gait performance is linked to co-morbidity, risk of falling [5], disability and mortality [6]. Given that people with ID already experience lifelong levels of low physical activity $[7,8]$, identifying and treating possible gait abnormalities to keep them active as long as possible is of utmost importance.

ID might originate from a range of different causes being either genetic (e.g. Down Syndrome, PraderWilli syndrome, Williams syndrome) or acquired (e.g. cerebral palsy, herpes encephalitis, lead intoxication). Delayed motor development is often seen in the population with ID, certainly at a younger age. A delayed onset of walking, after the age of 16 months, is common and occurs in 1 out of 5 children with ID [9]. Children with borderline intellectual function and mild ID score below the norm on motor function tests [10-13]. Children with more severe ID show even poorer motor performance [10-12]. Both in typically developing (TD) and ID populations, significant correlations have been found between motor performance and cognitive functions such as IQ, executive function and language development [12-14] with stronger relations between developmental domains in ID[13]. In populations with mild and borderline ID, 19 to $23 \%$ of the variance in motor functions can be explained by cognition $[10,12]$. The neuroanatomical theory of common pathways explaining the 
coupling between motor function and cognition is supported by both behavioral research and central imaging $[15,16]$. Motor control and cognition share common pathways in the dorsolateral prefrontal cortex, cerebellum and connecting structures including the basal ganglia $[10,13]$. The strong coupling between the different developmental domains in the ID population might thus be related to a higher incidence of brain anomalies and an atypical function of the brain (i.e. atypical brain development concept of Kaplan)[11, 13].

The role of cognition in gait has to be recognized. Cognitive functions such as integration of attention, planning, memory and perception all play an important role [17]. As such, gait abnormalities can be expected in individuals with ID. Nevertheless, several patients with ID, especially with underlying genetic cause, also show physical abnormalities that might affect their gait pattern. In order to treat potential gait abnormalities in patients with ID, a better understanding of the contribution of physical features and cognitive components is necessary. In 2014, Almuhtaseb and co-workers [18] performed a systematic literature review on gait characteristics in individuals with ID. They showed that gait abnormalities are evident in the ID population, both in people with genetic syndromes and with acquired ID. Physical characteristics, such as hypermobility, ligament laxity and muscle hypotonia in Down syndrome or severe obesity in Prader-Willi syndrome had a considerable biomechanical effect on the gait pattern. However, the specific physical features do not explain all of the gait abnormalities in the ID population. To better understand this, the relation between gait and cognition deserves attention.

The overall aim of this narrative review is to provide the reader with an overview of the gait features that are common in a population with ID as well as discuss potential benefits and challenges of performing instrumented gait analysis in patients with ID. As such this review consists of three main parts. First we will provide an update of the literature [18] to identify gait abnormalities common in populations with ID and formulate a hypothesis on the link between cognition and gait; second we will discuss the standard gait analysis protocol with its benefits and challenges; third we will present a case report of gait abnormalities in a patient with severe ID due to a genetic cause (syndrome of Dravet) and link this to information from literature. 


\section{CENTRAL BODY}

\section{Gait abnormalities in patients with an intellectual disability}

An update of the systematic review by Almuhtaseb [18] was performed using the same search strategy. The search query [(intellectual disability) AND gait] was entered in Pubmed on May $3^{\text {rd }}$, 2018 and publication date limits were set starting from August $1^{\text {st }}, 2013$ (table1). Two researchers (A.H. and L.W.) independently screened the citations on title and abstract according to predetermined criteria. The screening results were discussed until consensus was found and selected articles were subjected to screening on full text using the same criteria. Articles were included when the population (P) consisted of participants with ID of any age, when the primary outcome (O) described gait characteristics in terms of spatio-temporal parameters, kinematics, kinetics and/or dynamic electromyography collected during overground and unperturbed locomotion (I) and when the study (S) contained original research published in English, Dutch, French or German. Occasional and subjective reports on gait deviations in merely genetical or pharmacological research and case reports were excluded.

Two researchers (A.H. and L.W.) extracted the following data using a structured form: study characteristics (aim and design), population (diagnosis, inclusion and exclusion criteria and participant characteristics), measurement equipment and protocol, statistical analysis, outcome measures and results.

The search yielded 189 articles that were published since August $1^{\text {st }}, 2013$. After screening on title and abstract, 155 articles were excluded. The remaining 34 articles were screened on full text and another 14 studies were excluded for not meeting the selection criteria. As such, 20 studies were included in this review, which can be considered an update of the literature review on gait characteristics in the ID population, published by Almuhtaseb [18] (Fig. 1)

\subsection{Descriptive information of the studies included}

Twelve studies had a case-control design [19-30], four were cross-sectional studies [31-34], two were cohort studies [35, 36] and two were randomized controlled trials [37, 38]. 
Twelve studies investigated syndrome-specific ID populations, of which five were Down syndrome (DS) [21-25, 29], two were Prader - Willi syndrome (PWS) [27, 35], one Cri du Chat syndrome (CDC) [19], one Fragile - X associated tremor/ataxia syndrome (FXAT+) [39], one Rett syndrome (RS) [36], one Rubinstein - Taybi syndrome (RTS) [20] and one Williams syndrome (WS) [24]. Three studies combined various etiologies such as Down syndrome with other unknown syndromes [37, 38] or Down syndrome with autism spectrum disorder and pervasive developmental disorders [26]. In five remaining studies, the etiology of the ID was not specified [30-34]. Twelve studies provided information on the level of ID (Table 2).

The studies covered a wide range of ages from 3 till 80 years of age. Sample sizes varied between 10 and 98 subjects. All of the included studies reported on overground walking and all except one [30] explicitly stated that this was performed at preferred speed. In seven studies this was performed barefoot [21-23, 27, 34, 35, 37], one study reported that subjects wore regular footwear [38]. The other studies provided no information on this topic (

\subsection{Spatio-temporal gait parameters}

Sixteen studies provided data on spatio-temporal parameters using different methods. The GAITRite® electronic walkway was most often used $[19,24,25,31,34,36,37]$. A 3D video motion system (either Vicon or Elite BTS) was also frequently used [20, 21, 27, 29, 35]. Three studies used inertial sensor technology [26, 30, 39] while the remaining study measured gait speed by means of a chronometer during a 10 meter walk test [38].

In Down syndrome, compared with a control group, three studies [21, 24, 29] reported a significantly lower gait speed (DS: $0.66-1.05 \mathrm{~m} / \mathrm{s}$ vs. control group CG: $1.10-1.30 \mathrm{~m} / \mathrm{s}$ ) together with a lower cadence (DS: 97 - 103 steps/min vs. CG: 109 - 115 steps/min) and a shorter step length (DS: 0.42 $0.59 \mathrm{~m}$ vs. CG: $0.59-0.71 \mathrm{~m})$. However, one study [25] contradicts these findings reporting a higher gait speed, a higher cadence and a longer step length in Down syndrome compared to controls (Appendix 1, Table 1). In Prader - Willi syndrome, similarly, gait speed is significantly reduced (PWS: $0.88-0.98 \mathrm{~m} / \mathrm{s}$ vs. CG: $1.03-1.20 \mathrm{~m} / \mathrm{s}$ ), cadence is decreased (PWS: 105 steps/min vs. CG: 116 steps/min) and step length is significantly shorter (PWS: $0.51 \mathrm{~m}$ vs. CG: $0.63 \mathrm{~m}$ ) compared to a 
control group [27, 35]. In other syndrome-specific ID, except for Rubinstein - Taybi syndrome [20], similar trends of low gait speed [19, 36, 39], low cadence [39] and short step length [19] exist. In Cri $d u$ Chat syndrome [19], step width is significantly increased (CDC: $11.2 \mathrm{~cm}$ vs. CG: $8.5 \mathrm{~cm}$ ). In patients with Fragile-X associated tremor/ataxia, duration of swing is decreased and duration of double support is significantly increased [39] (Appendix 1, Table 1).

In a general population with ID, either spatio-temporal parameters of gait did not show significant differences compared to a control group $[26,30]$ or no control group was available making it difficult to compare $[31,34,37,38]$.

\subsection{Gait variability}

Four studies report gait variability data either as coefficients of variation [20, 24, 39] or intra-subject standard deviations [32].

In syndrome-specific ID populations, coefficients of variation for step length [20], stride length [20, 39], cadence [20, 39], step time [24], stride time [20], step width [20] and gait speed [24, 39] are significantly larger compared to a control group. Oppewal [32] reported intra-subject standard deviations for spatio-temporal parameters in a general ID population, but this study did not have a control group, which makes it difficult to compare. Nevertheless, intra-individual variation in step width appears to be large (Appendix 1: Table 2).

\subsection{Kinematic gait parameters}

Three studies report kinematic data of which two are performed in Down syndrome $[21,23]$ and one in Prader - Willi syndrome [35]. All studies use a 3D video motion system with either the Davis marker set-up [21, 23] or its adjusted version in Plug-in Gait [35].

In Down syndrome, compared to a control group, higher values are found for hip flexion at initial contact while hip extension in stance and hip range of motion in the sagittal plane are reduced [21]. Knee extension in stance, maximal knee flexion in swing and knee range of motion in the sagittal plane are also reduced. At initial contact, the ankle is in plantar flexion instead of neutral [21], there is less ankle dorsiflexion in stance as well as reduced ankle plantar flexion at push-off and ankle range of 
motion in the sagittal plane [21]. The mean foot progression angle is significantly more external [23]. (Appendix 1: Table 3)

In Prader - Willi syndrome, differences are found around the ankle joint and to a lesser degree at the knee. There is a reduction in ankle plantar flexion at push-off and in ankle range of motion in the sagittal plane while knee flexion at initial contact is increased [35]. (Appendix 1: Table 3)

\subsection{Kinetic gait parameters}

Three studies report on kinetic data in Down syndrome [21, 22] and Prader-Willi syndrome [35]. In both populations only net joint moments and powers around the ankle are reported. Maximal ankle plantar flexion moment (DS: $0.99(0.17)-1.08(0.38) \mathrm{Nm} / \mathrm{kg}$; PWS: $1.02(0.17) \mathrm{Nm} / \mathrm{kg}$; CG: 1.29 $(0.23)-1.49(0.25) \mathrm{Nm} / \mathrm{kg})$ and maximal power generation at push-off (DS: $1.59(0.96)-1.60(0.65)$ W/kg; PWS: 1.59 (0.51) W/kg; CG: $3.01(0.52)-3.73(0.71) \mathrm{W} / \mathrm{kg})$ are significantly reduced in both syndrome-specific ID populations. Since kinetic gait parameters are largely dependent upon walking speed, Cimolin [35] normalized maximal ankle joint power for speed and still found significantly smaller normalized ankle joint power in Prader-Willi syndrome compared to controls (PWS: 1.82 (0.55) W.s/kg.m; CG: 2.95 (0.80) W.s/kg.m).

\subsection{Discussion and hypothesis generation}

The results show significant gait deviations in the ID population with an underlying genetic syndrome. Main features are a reduced gait speed, short step length, increased step to step variability and a gait pattern with increased flexion in the knee joint and/or hip joint together with decreased ranges of motion at the ankle joint during the second and third rocker. Kinematic differences observed around the ankle joint coincide with reduced force and power generation at push-off. These observations are in line with the findings of Almuhtaseb [18].

In the general ID population however gait deviations are much less evident which contradicts the findings of Almuhtaseb[18]. In her review she concluded that gait in the general ID population is also characterized by low gait speed and/or short step or stride length. Looking closer at the included studies, ID ranges from moderate to profound while in the studies included in this review ID in the 
general population is mostly mild to moderate (Appendix 1: Table 1). This might be a possible explanation for the observed differences.

Observed gait deviations in the ID population might be attributed to either physical characteristics or cognitive components. Physical characteristics are addressed in four studies looking at obesity in Prader-Willi syndrome [35] and Down syndrome [21] and looking at foot deformities in Down syndrome $[22,23]$. While these physical characteristics indeed have an effect on the gait, they do not seem to be the only determining factor. Cimolin [35] found significant differences in gait pattern between Prader-Willi syndrome and the obese control group as well. Similarly, in the studies of Galli $[22,23]$ also the group with normal to high arch feet showed significant alterations in gait pattern, although less pronounced than in the flat feet group. (Appendix 1: Table 2 and 3) Therefore, while physical characteristics remain important, other factors such as the role of cognition cannot be ruled out.

The cognitive components in relation to gait are best studied using dual task paradigms. While data extraction focused on unperturbed walking, several studied included a dual-task paradigm [19, 24, 25, 31]. Results showed clear competition between the motor task (gait) and the dual task, whereby carrying out a secondary task will degrade motor performance to a larger extent in the ID group than in the healthy control group. (Appendix 2) Nevertheless, performance is dependent upon the kind of task and the population under investigation. For example, in Down syndrome, gait is mostly affected when the secondary task addresses the executive working memory while patients with Williams syndrome are more affected by tasks requiring set-shifting and visual-spatial processing [24]. Nevertheless it is clear that in ID populations, increased attentional resources are required during overground locomotion. The hypothesis has been formulated that gait is not a learned and preprogrammed motor task in these populations [25]. This hypothesis can be considered from the viewpoint of the atypical brain development concept affecting both cognitive and motor functions. We hypothesize that due to brain or brain function anomalies, control of gait has remained largely immature requiring more attentional resources. Possibly, this immature control is partially responsible 
for the observed immature features such as short and wide steps, increased variability and a flexed position of the lower limbs [40, 41].

\section{Protocol development.}

Several gait analysis methods have been developed and applied in clinical practice. Depending on the outcome of interest, a variety of measuring devices are selected to develop gait analysis protocols. When spatio-temporal parameters and gait variability are the only outcomes needed, electronic walkways or inertial sensors are feasible. Electronic walkways such as the GAITRite ${ }^{\circledR}$ can provide quick and accurate measurements and are suitable for children with motor disorders [42]. Inertial sensors enable evaluation of a large number of steps outside of a hospital or research laboratory setting. Although spatio-temporal parameters are relevant to detect functional deviations in patients' gait, they do not provide further information on body motion and muscle function. This information however is often necessary to evaluate and treat gait problems and can be measured using adequate methods of body motion analysis during gait. Two general categories of such gait analysis methods exist: quantitative measurements using instrumented gait analysis (IGA, also referred to as three dimensional gait analysis) and observational assessments using video gait analysis (VGA). In the first category, three types of devices are used: image processing tools, floor sensors and wearable sensors, extensively described in literature [43].

To obtain valid and reliable data through gait analysis, a standardized measurement protocol is essential $[44,45]$. The use of gait analysis in children with ID is not common and standard protocols may be challenging for this population. Therefore in this section we will provide an overview of established IGA and VGA protocols and discuss their benefits and challenges in this population.

\subsection{General gait analysis protocol}

Standard gait analysis protocols consist of the same basic elements. Recordings start with a static trial which provides information on the standing posture of the patient and allows calibration of the marker model in instrumented gait analysis. Afterwards, dynamic overground walking trials are performed on a sufficiently long walkway. The patient should walk at self-selected walking velocity in a manner that 
is representative for their usual gait. Instructions on how to walk should be avoided in order to evoke a spontaneous pattern, but corrections are made when the child starts to show an undesirable gait such as running or excessive looking around and marching in a funny way. Gait analysis sessions should include structured physical examination for joint range of motion, muscle length, muscle strength and selectivity, spasticity and other clinical features needed for thorough interpretation of the gait analysis data [2].

\subsection{Instrumented gait analysis}

A general consensus on optimal IGA methods exists [44] and detailed description lies beyond the scope of this review. In our centre, the gait laboratory (Multidisciplinary Motor Center Antwerp, $\mathrm{M}^{2}$ OCEAN) is equipped with eight Vicon T10 cameras (100fps, 1 Megapix), Vicon Nexus, Bodybuilder and Polygon software, three AMTI type OR 6-7 force plates (1000fps), one Accugait force plate (1000 fps) and a 16 channel telemetric wireless EMG system (Aurion Zerowire). Retroflective markers are placed on bony landmarks following the PlugInGait Lower Limb marker model, which enables the quantification of pelvic position as well as hip, knee and ankle joint angles in all three anatomic planes. The lower limb model is preferred over full body models to reduce the number of markers, as these are experienced as disturbing by many children with ID. The combination of the optoelectronic tracking system and force plates allows the calculation of spatio-temporal data and the quantification of body segment and joint movements (kinematics) combined with the forces that cause these movements (kinetics), the core measurements of IGA. Simultaneous video registration provides a visual control and quality check. Additional measurement modalities can be added into an extended protocol if desired and achievable, as discussed further on and presented in table 3. Integrated dynamic EMG recordings allow the analysis of muscle activation patterns during walking. The preparation of the skin and application of electrodes takes more time and is uncomfortable for some children with ID. Therefore EMG recordings are left out of the core protocol to reduce the burden for the patient. Foot plantar pressure distribution measurement systems are additionally available for specific purposes. 
Walking trials are repeated until at least three representative strides for each leg are recorded. A "clean" foot strike on a force plate is required for the collection of kinetic data. Targeting force plates is not representative for usual gait, therefore the child's attention should not be drawn towards the force plates. In children who are less cooperative, it may not be possible to repeat trials until all selected strides contain reliable kinetic data. For children who make too small or shuffling steps, clean foot strikes on force plates are impossible. The collection of reliable kinematic data is prioritized over kinetic data. Kinetic data are only computed in case reliable foot strikes on force plates occurred. In case a child usually walks with orthotic devices, static and dynamic recordings are repeated with the child wearing their devices using the same marker placement.

The collected data are processed afterwards to provide data in an appropriate format for clinical interpretation. Spatio-temporal parameters, kinematic and kinetic time profiles and, if available, muscle activation patterns are reported. Age-related reference values of typically developing children (mean +/- 1SD) are provided for comparison. Consistency plots, containing kinematic and kinetic data for up to six trials of the same side, are presented to assess the level of variability within the child's gait pattern. In case there is general variability across all graphs with an average trial to trial variability above $20^{\circ}$, caution is needed for the interpretation, as isolated gait trials are less representative for the child's overall gait pattern. Comparison reports are made in which barefoot trials and trials with shoes and orthotic devices are plotted together to evaluate the impact of the device on the gait pattern. Similarly, trials of consecutive sessions of the same child are brought together to assess the evolution of gait problems over time.

The most important strengths of IGA are its high reliability and accuracy, making it the gold standard for gait analysis. In their systematic review, McGinley et al. [4] found that intra-rater reliability indices were typically higher than 0.80 in the sagittal plane (except for pelvic tilt), and slightly lower in the coronal and transversal plane $\left(>0.70\right.$ and $<0.70$ respectively). Measurement errors were lower than $4^{\circ}$ in the sagittal plane and around $2^{\circ}$ in the coronal plane, smaller than what is considered a clinically important difference [4]. Moreover, Kawamura et al. proved that IGA is significantly more accurate in the detection of gait deviations than comparable VGA [46]. 


\subsection{Video gait analysis}

Limited studies have proposed VGA protocols as an accessible alternative to IGA [47] and recording methods are less extensively discussed. In our VGA protocol, two video cameras are positioned next to the walkway: one perpendicular and one parallel to the line of progression, providing sagittal and coronal plane observations respectively. A top view camera could additionally provide transverse plane images, but visibility in this plane is especially deprived. Two extra cameras can be added, zooming in on the patient's feet to provide close-up images (table 3).

Various observational gait assessment tools have been established to standardise the interpretation of VGA data in paediatric populations $[45,47]$. The best results on reliability and validity assessments were found for the Edinburgh Visual Gait Score (EVGS)[47]. This tool evaluates trunk, pelvis, hip, knee and ankle in the three anatomical planes using a three-point ordinal scale. However, observational gait assessment tools are highly dependent on the experience of the rater [48] and intrarater reliability is lower than in IGA $(0.25$ to 0.79$)$. Furthermore, these tools were all designed and validated in populations of children with $\mathrm{CP}$ and further research on their applicability in children with ID is needed.

The strength of VGA is its accessibility because of the low cost and user friendliness, both for the patient and the assessor. VGA provides an opportunity for more subjective qualitative description of the gait pattern. Also quantitative estimates of joint angles at specific points of the gait cycle can be made using goniometry. Custom-made software is available for this purpose [49]. However, these estimates are prone to projection errors and are less reliable than IGA. Video-based markerless motion capture systems are being developed, but more research is needed to improve their accuracy and applicability in clinical gait analysis [39].

\subsection{Considerations}

The choice of adequate method and measurement modalities depends on the clinical goal, the available resources and the abilities of the patient. 
The main goals of clinical gait assessment are the identification of gait deviations and the evaluation of their progress over time, in order to optimize the treatment plan. VGA may be sufficient to perform early follow-up of gait development and detect potential delay. Nevertheless, IGA is more adequate in detecting primary problems and compensatory strategies. VGA already offers insight in gait pathology and is helpful to establish a basic treatment plan. However, IGA is advised to guide clinical decision making and follow up after intervention when more complex treatment options are considered such as orthotic devices, orthopaedic surgery or botulinum toxin injections [50].

Very decisive in selecting the adequate protocol are the available resources. Not only does IGA require a fully equipped laboratory, its accuracy is also highly dependent on the experience of the staff that operates it and the processing and interpretation of IGA data requires training [3]. Therefore VGA may offer a more user friendly and low cost alternative when these requirements are not met.

In a population of children with ID, the argument of the abilities of the child becomes especially important. Standard IGA procedures take up to two hours and demand a certain level of cooperation of the patient. If a child with behavioural problems is not able to sit still during preparations (electrode and marker placement) or if they pull the markers off, no reliable data can be obtained. In such cases, VGA is probably more appropriate. Furthermore, a child should be able to carry out simple instructions to walk in a straight line in a representative way, which might be easier in the less distractive setting of VGA than in IGA. The extensiveness of the protocol of IGA influences the duration of a session and thus the burden for the patient. Within a reduced or extended protocol, duration of a session will further depend on the number of trials collected to assess consistency, the number of attempts needed to obtain clean foot strikes and the number of different situations (barefoot, orthotics, walking aids) that need to be analysed.

\section{Case example of a patient with Dravet Syndrome.}

Dravet Syndrome is a rare and severe form of drug resistant epilepsy and developmental delay with intellectual disability and behavioral problems [51] caused by a genetic mutation in SCN1A. A variety of gait characteristics have been reported in literature with crouch gait being the most observed gait 
pattern [52, 53]. Other observed patterns include parkinsonian and cerebellar gait [54]. The cause of crouch gait is multifactorial and may include muscle weakness, spasticity, contractures or lever arm dysfunction [55]. Clinical examination findings on these factors were inconsistent in patients with Dravet syndrome.

This case study presents a girl, diagnosed with Dravet Syndrome, who consulted our facility at the ages of $4 \mathrm{y} 4 \mathrm{~m}, 6 \mathrm{y} 0 \mathrm{~m}, 7 \mathrm{y} 2 \mathrm{~m}$ and $8 \mathrm{y} 2 \mathrm{~m}$ for instrumented gait analysis, walking barefoot without aids (See Appendix 3: Polygon Viewer). Due to young age and limited cooperation an extended instrumented protocol was not possible in every session and high quality trials were sometimes difficult to collect (in Figure 2 a trial with artefact had to be used due to lack of high quality trials).

\subsection{Patient history, developmental assessment and physical examination}

Patient history showed near normal development during the first year of life but a rapid delay thereafter. The milestone of independent sitting was acquired at the age of 7 months. Developmental age at 12 months was assessed with the Bailey Scales of Infant Development - II (BSID-II-NL) and was normal. However, a strong delay was observed in acquiring the milestone of independent walking, at an age of 36 months. At the age of 26 months, developmental age was only 15 months (BSID-IINL, cognitive subscale) and also motor developmental age was delayed reaching only 12 months (BSID-II-NL, motor subscale).

A standardized physical examination was performed at the ages of $6 y 0 \mathrm{~m}, 7 \mathrm{y} 2 \mathrm{~m}$ and $8 \mathrm{y} 2 \mathrm{~m}$. The most obvious finding is a severe pes planovalgus of both the left and right foot, already at the age of 6y0m, for which insoles were prescribed. Starting at the age of $7 \mathrm{y} 2 \mathrm{~m}$ internal rotation of the hips in stance is observed, becoming more severe at the age of $8 \mathrm{y} 2 \mathrm{~m}$ leading to "kissing knees". Femoral anteversion is slightly increased, especially at the left side $\left(20^{\circ}\right.$ at age $6 \mathrm{y} 0 \mathrm{~m}$ going up to $40^{\circ}$ at age $\left.8 \mathrm{y} 2 \mathrm{~m}\right)$. Hamstrings are fairly short (popliteal angle deficit of $30^{\circ}(\mathrm{L}, \mathrm{R})$ at age $6 \mathrm{y} 0 \mathrm{~m}$ going up to $35^{\circ}(\mathrm{L})$ and $45^{\circ}(\mathrm{R})$ at age $8 \mathrm{y} 2 \mathrm{~m}$ ) while calf muscles appear elongated (large passive ankle dorsiflexion range of motion of $15^{\circ}-20^{\circ}$ with the knee extended). 


\subsection{Spatio-temporal analysis}

Dimensionless gait speed was highly variable within and between all sessions ranging from reduced (>2SD with reference to age-related TD children) at the age of $7 \mathrm{y} 2 \mathrm{~m}(0.24 \pm 0.05)$, over slightly reduced ( $>1 \mathrm{SD}$ with reference to age-related TD children) at the age of $4 \mathrm{y} 4 \mathrm{~m}(0.29 \pm 0.07)$ and $8 \mathrm{y} 2 \mathrm{~m}$ $(0.37 \pm 0.04 \mathrm{~s})$ to normal at the age of $6 \mathrm{y} 0 \mathrm{~m}(0.48 \pm 0.05)$. This is in line with literature where reduced to normal gait speed and high variability is reported in patients with ID [18, 21, 24, 29]. Dimensionless step length is reduced in three out of four sessions $(0.50 \pm 0.09-0.64 \pm 0.08 \times$ leg length $)$ with more variability than in TD children. Only at $6 \mathrm{y} 0 \mathrm{~m}$ dimensionless step length $(0.77 \pm 0.10 \mathrm{x}$ leg length) was within normal values. A high to slightly increased cadence is seen at ages of $4 \mathrm{y} 4 \mathrm{~m}$ and $6 \mathrm{y} 0 \mathrm{~m}(152 \pm 27$ steps $/ \mathrm{min}$ and $158 \pm 7 \mathrm{steps} / \mathrm{min}$ ) with very high variability at $4 \mathrm{y} 4 \mathrm{~m}$, where at $7 \mathrm{y} 2 \mathrm{~m}$ cadence is slightly reduced $(112 \pm 12 \mathrm{steps} / \mathrm{min})$ and at $8 \mathrm{y} 2 \mathrm{~m}$ it is within normal values $(131 \pm 8 \mathrm{steps} / \mathrm{min})$. This high cadence is in contradiction with literature $[21,28,35]$ and might indicate that at a young age, she is still able to compensate for the short step length in order to maintain a functional gait speed.

\subsection{Kinematics}

At the hip (Figure 2; Appendix 3) normal to increased flexion at IC and slightly reduced extension at terminal stance is seen at all sessions with high variability within sessions, a typical feature of ID gait [18]. Due to an increased hip flexion during swing, this does not lead to a reduced sagittal hip ROM.

At the knee, increased knee flexion (range $12-26^{\circ}$ ) at IC contact was seen at all occasions. Normal shock absorption (increased knee flexion during loading response) was present in most of the trials. At terminal stance knee extension was reduced in most trials and normal in very few trials (range $5-25^{\circ}$ ), again in line with literature and variable within each session.

At the ankle (Figure 2; Appendix 3), in contrast to literature on gait in patients with ID, where a plantar flexed IC was often observed, a neutral position at IC was seen at all sessions. However, subsequent plantar flexion during first rocker was often absent. Second rocker showed highly variable patterns over all sessions, sometimes with a lack of tibia progression (horizontal), sometimes with plantar flexion (reversed second rocker) and sometimes with increased dorsiflexion. Push off was mostly normal but at the age of $8 \mathrm{y} 2 \mathrm{~m}$, a decreased ROM during push off was observed (Figure $3 \mathrm{C}$ ). 
During swing, often a plantar flexion was seen at the end of swing that affected foot clearance only in few occasions.

Foot progression angle (Figure 2; Appendix 3), was again highly variable, but more often increased externally than increased internally rotated.

\subsection{Kinetics}

Collection of valid kinetics is a challenge in children with ID. Nevertheless, we were able to collect at least one trail per side per session.

Internal net joint extension moment of hip and knee was decreased at terminal stance in all four sessions. At the ankle, internal net joint plantar flexion moment was consistently decreased and ankle plantar flexion power at push off was only half of normal values in all trails in all sessions (Figure 3; Appendix 3).

\subsection{Muscle activation patterns}

EMG collection was difficult in this child and muscle activation patterns could only be evaluated at the ages of $6 y 0 \mathrm{~m}$ and $7 \mathrm{y} 2 \mathrm{~m}$. Prolonged activity during midstance is observed in the medial and lateral hamstrings as well as in the $\mathrm{m}$. Rectus femoris and $\mathrm{m}$. Vastus lateralis leading to co-contraction. Distally, gastrocnemius muscle is active too early in stance, leading to co-contraction with $\mathrm{m}$. Tibialis anterior.

\subsection{Clinical implications}

Kinematic and kinetic data suggest the progressive development of a crouch gait pattern. The occurrence of hamstrings shortening is of concern as well as the increasing internal rotation of the hips. The interaction between these physical characteristics and the deviant gait pattern might lead to a vicious circle of increasing crouch. Crouch gait is a severe gait deviation that, due to the high amount of muscle work required, is very exhausting and can dramatically limit mobility. Therefore, at the age of $7 \mathrm{y} 2 \mathrm{~m}$ a rigid ankle-foot orthosis was prescribed to enhance correct knee alignment at initial contact. At first re-evaluation, indeed, knee angle at initial contact was less flexed when walking with the ankle-foot orthosis than walking barefoot. On the other hand, ankle motion is limited and power 
generation at push off might be even further compromised. Long term follow-up is necessary to evaluate the potential benefits and risks of this orthotic intervention.

\section{DISCUSSION}

The aim of this review was to establish an overview of the gait features that are common in a population with ID as well as discuss potential benefits and challenges of performing instrumented gait analysis in patients with ID. For this purpose, an update of a previously published literature review was performed [18]. In accordance with the original search strategy, this was limited to only one database, i.e. Pubmed, possibly reducing the comprehensiveness of the results.

In a population with ID we need to be aware that both cognitive and physical components can contribute to a deviant gait pattern. The usefulness of a clinical gait analysis in this population is that it allows clinicians to objectify the relationship between physical characteristics and gait features $[2,3]$. While it is much more difficult to amend to gait deviations arising from poor cognition, physical characteristics are amenable to treatment. To do so, information on kinematics of gait, and preferably also information on kinetics of gait and muscle function is required.

In our case example, significant gait deviations were found that are also reported in the literature on the ID population with genetic syndromes [18], such as a reduced gait speed, decreased step length, increased step to step variability [19-21, 24, 27-29, 33, 35] and increased flexion in the knee and hip joint along with reduced moments and power generation at push-off. Despite its challenges, gait analysis contributed to clinical decision making in this child, i.e. the prescription of ankle foot orthoses which led to improved extension in stance. It cannot be ruled out that cognition $[11,13]$ and competition for attentional resources [24] as well as motivational aspects also played an important role in determining the gait pattern as the flexed gait pattern observed in this child is also frequently observed in other populations with ID such as Prader-Willi syndrome [35] and Down syndrome [21]. But on top of these common features of ID gait, clinical gait analysis pointed towards rotational deformities and muscular contractures that appeared to worsen with increasing age. A mutual interaction between these physical characteristics and the flexed gait pattern is plausible and concern is 
that the child would enter a vicious circle of increased flexion during gait, leading to crouch gait, and worsening rotational deformities. Although at an early age it was virtually impossible to distinguish cognitive from physical components affecting gait, follow-up through clinical gait analysis showed that physical components came to the forefront when the child grew older and warranted treatment.

As mentioned before, obtaining a good quality IGA in children with ID is a challenge. Sometimes, this results in less reliable data due to difficult marker placement and/or difficult behavior. The recorded gait pattern should represent gait in daily life but in children with behavioral problems and low IQ, the lab setting can largely affect their gait. This can be further aggravated by attaching markers and electrodes to their skin. Furthermore, touching of markers may lead to artefacts. Also clear strikes on the force plate are not easy to obtain. Sometimes a child performs 20 or more gait trials and we only obtain good kinetic data in one.

In extremely challenging cases, or in young children, it might therefore be wise to opt for a standardized VGA protocol. Benefits of the VGA protocol are the low cost and the user friendliness. Duration of a session is dramatically reduced which promotes the cooperation of children with difficult behavior. While VGA does not provide the accuracy and reliability of IGA, it is often sufficient to detect whether gait deviations exist and follow their progression over time. In case severe deteriorations in gait or in physical characteristics are observed, an IGA can be considered. When a child is already familiar with the lab setting from previous VGA, improved cooperation and better quality IGA are expected. With our case example we have shown that, although challenging, good quality data can be obtained.

While not easily amenable to treatment, it might be interesting to obtain information on the cognitive load required to walk. This can be done by dual task paradigm in which case spatio-temporal parameters are considered as primary outcome measures. As discussed before, spatio-temporal parameters provide information on the functionality of the gait pattern and thereby (partially) reflect functioning in daily life. In these cases, use of gait mats (e.g. GaitRite ${ }^{\circledR}$ ) or inertial sensors (with accompanying software e.g. MoveMonitor and MoveTest, McRoberts), can be a good choice because of their limited processing time and direct availability of data. 


\section{CONCLUSIONS}

Gait analysis has substantial value for patients with ID. In this population, both cognitive and physical components can contribute to a deviant gait pattern. Gait analysis allows clinicians to objectify the relationship between physical characteristics and gait features. The choice of adequate method and measurement modalities, being VGA or IGA, should depend on the clinical goal, the available resources and the abilities of the patient.

\section{REFERENCES}

[1] J.R. Gage, P. Deluca, T. Renshaw, Gait analysis: principles and applications, J.Bone Joint Surg. 77A(10) (1995) 1607-1623. Ann Hallemans.

[2] K. Desloovere, G. Molenaers, H. Feys, C. Huenaerts, B. Callewaert, P. Van de Walle, Do dynamic and static clinical measurements correlate with gait analysis parameters in children with cerebral palsy?, Gait Posture 24(3) (2006) 302-13. https://www.ncbi.nlm.nih.gov/pubmed/16303305.

[3] R. Baker, A. Esquenazi, M.G. Benedetti, K. Desloovere, Gait analysis: clinical facts, European journal of physical and rehabilitation medicine 52 (2016) 560-574.

[4] J.L. McGinley, R. Baker, R. Wolfe, M.E. Morris, The reliability of three-dimensional kinematic gait measurements: a systematic review, Gait Posture 29(3) (2009) 360-9. https://www.ncbi.nlm.nih.gov/pubmed/19013070.

[5] M.L. Callisaya, L. Blizzard, M.D. Schmidt, J.L. McGinley, V.K. Srikanth, Ageing and gait variability--a population-based study of older people, Age and ageing 39(2) (2010) 191-7.

[6] A.F. Ambrose, G. Paul, J.M. Hausdorff, Risk factors for falls among older adults: a review of the literature, Maturitas 75(1) (2013) 51-61. http://www.ncbi.nlm.nih.gov/pubmed/23523272.

[7] A. Oppewal, T.I. Hilgenkamp, R. van Wijck, J.D. Schoufour, H.M. Evenhuis, Physical fitness is predictive for a decline in the ability to perform instrumental activities of daily living in older adults with intellectual disabilities: Results of the HA-ID study, Res Dev Disabil 41-42 (2015) 76-85.

[8] A. Oppewal, T.I. Hilgenkamp, R. van Wijck, J.D. Schoufour, H.M. Evenhuis, Physical fitness is predictive for a decline in daily functioning in older adults with intellectual disabilities: results of the HA-ID study, Res Dev Disabil 35(10) (2014) 2299-315.

[9] S.L. Bishop, A. Thurm, C. Farmer, C. Lord, Autism Spectrum Disorder, Intellectual Disability, and Delayed Walking, Pediatrics 137(3) (2016) e20152959.

[10] E. Hartman, S. Houwen, E. Scherder, C. Visscher, On the relationship between motor performance and executive functioning in children with intellectual disabilities, Journal of intellectual disability research : JIDR 54(5) (2010) 468-77.

[11] P.J. Vuijk, E. Hartman, E. Scherder, C. Visscher, Motor performance of children with mild intellectual disability and borderline intellectual functioning, Journal of intellectual disability research : JIDR 54(11) (2010) 955-65.

[12] B. Smits-Engelsman, E.L. Hill, The relationship between motor coordination and intelligence across the IQ range, Pediatrics 130(4) (2012) e950-6.

[13] S. Houwen, L. Visser, A. van der Putten, C. Vlaskamp, The interrelationships between motor, cognitive, and language development in children with and without intellectual and developmental disabilities, Res Dev Disabil 53-54 (2016) 19-31.

[14] H. Kim, A.G. Carlson, T.W. Curby, A. Winsler, Relations among motor, social, and cognitive skills in pre-kindergarten children with developmental disabilities, Res Dev Disabil 53-54 (2016) 43-60. 
[15] M.N. Spann, R. Bansal, T.S. Rosen, B.S. Peterson, Morphological features of the neonatal brain support development of subsequent cognitive, language, and motor abilities, Human brain mapping 35(9) (2014) 4459-74.

[16] C. Dockstader, W. Gaetz, C. Rockel, D.J. Mabbott, White matter maturation in visual and motor areas predicts the latency of visual activation in children, Human brain mapping 33(1) (2012) 179-91.

[17] V.J. Verlinden, J.N. van der Geest, A. Hofman, M.A. Ikram, Cognition and gait show a distinct pattern of association in the general population, Alzheimer's \& dementia : the journal of the Alzheimer's Association 10(3) (2014) 328-35.

[18] S. Almuhtaseb, A. Oppewal, T.I. Hilgenkamp, Gait characteristics in individuals with intellectual disabilities: a literature review, Res Dev Disabil 35(11) (2014) 2858-83. https://www.ncbi.nlm.nih.gov/pubmed/25105568.

[19] L.D. Abbruzzese, R. Salazar, M. Aubuchon, A.K. Rao, Temporal and spatial gait parameters in children with Cri du Chat Syndrome under single and dual task conditions, Gait Posture 50 (2016) 4752.

[20] J.R. Cazalets, E. Bestaven, E. Doat, M.P. Baudier, C. Gallot, A. Amestoy, et al., Evaluation of Motor Skills in Children with Rubinstein-Taybi Syndrome, Journal of autism and developmental disorders 47(11) (2017) 3321-3332.

[21] M. Galli, V. Cimolin, C. Rigoldi, C. Condoluci, G. Albertini, Effects of obesity on gait pattern in young individuals with Down syndrome, International journal of rehabilitation research. Internationale Zeitschrift fur Rehabilitationsforschung. Revue internationale de recherches de readaptation 38(1) (2015) 55-60.

[22] M. Galli, V. Cimolin, M. Pau, P. Costici, G. Albertini, Relationship between flat foot condition and gait pattern alterations in children with Down syndrome, Journal of intellectual disability research : JIDR 58(3) (2014) 269-76.

[23] M. Galli, V. Cimolin, C. Rigoldi, M. Pau, P. Costici, G. Albertini, The effects of low arched feet on foot rotation during gait in children with Down syndrome, J Intellect Disabil Res 58(8) (2014) 758-64. https://www.ncbi.nlm.nih.gov/pubmed/24750182.

[24] D.R. Hocking, J.C. Menant, H.E. Kirk, S. Lord, M.A. Porter, Gait profiles as indicators of domainspecific impairments in executive control across neurodevelopmental disorders, Res Dev Disabil 35(1) (2014) 203-14.

[25] M. Horvat, R. Croce, P. Tomporowski, M.C. Barna, The influence of dual-task conditions on movement in young adults with and without Down syndrome, Res Dev Disabil 34(10) (2013) 3517-25. [26] M. Iosa, D. Morelli, E. Nisi, C. Sorbara, S. Negrini, P. Gentili, et al., Assessment of upper body accelerations in young adults with intellectual disabilities while walking, running, and dual-task running, Hum Mov Sci 34 (2014) 187-95.

[27] D. Malatesta, L. Vismara, F. Menegoni, G. Grugni, P. Capodaglio, Effect of obesity onset on pendular energy transduction at spontaneous walking speed: Prader-Willi versus nonsyndromal obese individuals, Obesity (Silver Spring, Md.) 21(12) (2013) E586-91.

[28] J.A. O'Keefe, E.E. Robertson-Dick, D.A. Hall, E. Berry-Kravis, Gait and Functional Mobility Deficits in Fragile X-Associated Tremor/Ataxia Syndrome, Cerebellum (London, England) 15(4) (2016) 475-82.

[29] F. Salami, S.L. Vimercati, C. Rigoldi, A. Taebi, G. Albertini, M. Galli, Mechanical energy assessment of adult with Down syndrome during walking with obstacle avoidance, Res Dev Disabil 35(8) (2014) 1856-62.

[30] W.Y. Shieh, Y.Y. Ju, Y.C. Yu, C.K. Lin, Y.T. Lin, H.Y. Cheng, Stair-Walking Performance in Adolescents with Intellectual Disabilities, Sensors (Basel, Switzerland) 16(7) (2016).

[31] A. Oppewal, T.I.M. Hilgenkamp, The dual task effect on gait in adults with intellectual disabilities: is it predictive for falls?, Disabil Rehabil (2017) 1-7.

[32] A. Oppewal, D.A.M. Festen, T.I.M. Hilgenkamp, Gait Characteristics of Adults With Intellectual Disability, American journal on intellectual and developmental disabilities 123(3) (2018) 283-299.

[33] A. Oppewal, T.I.M. Hilgenkamp, The association between gait and physical fitness in adults with intellectual disabilities, Journal of intellectual disability research : JIDR 62(5) (2018) 454-466. 
[34] J. Salb, U. Lindemann, C. Woodward, S. Almutaseb, C. Becker, C. Sieber, et al., Gait Analysis in Adults with Intellectual Disabilities Living in a Residential Facility, Journal of applied research in intellectual disabilities : JARID 30(1) (2017) 205-210.

[35] V. Cimolin, L. Vismara, M. Galli, G. Grugni, N. Cau, P. Capodaglio, Gait strategy in genetically obese patients: a 7-year follow up, Res Dev Disabil 35(7) (2014) 1501-6.

[36] A. Djukic, R. Holtzer, S. Shinnar, H. Muzumdar, S.A. Rose, W. Mowrey, et al., Pharmacologic Treatment of Rett Syndrome With Glatiramer Acetate, Pediatric neurology 61 (2016) 51-7.

[37] K.J. Lee, M.M. Lee, D.C. Shin, S.H. Shin, C.H. Song, The effects of a balance exercise program for enhancement of gait function on temporal and spatial gait parameters in young people with intellectual disabilities, Journal of physical therapy science 26(4) (2014) 513-6.

[38] K. Lee, M. Lee, C. Song, Balance training improves postural balance, gait, and functional strength in adolescents with intellectual disabilities: Single-blinded, randomized clinical trial, Disability and health journal 9(3) (2016) 416-22.

[39] J.A. O'Keefe, A.A. Espinoza Orias, H. Khan, D.A. Hall, E. Berry-Kravis, M.A. Wimmer, Implementation of a markerless motion analysis method to quantify hyperkinesis in males with fragile $X$ syndrome, Gait Posture 39(2) (2014) 827-30. https://www.ncbi.nlm.nih.gov/pubmed/24252602.

[40] A. Hallemans, D. De Clercq, P. Aerts, Changes in 3D joint dynamics during the first 5 months after the onset of independent walking: A longitudinal follow-up study, Gait \& Posture 24(3) (2006) 270279.

[41] A. Hallemans, D. De Clercq, B. Otten, P. Aerts, 3D joint dynamics of walking in toddlers - A crosssectional study spanning the first rapid development phase of walking, Gait \& Posture 22(2) (2005) 107-118.

[42] V.C. Wondra, K. Pitetti, M.W. Beets, Motor Disabilities Using an Electronic Walkway System: Assessment of Reliability, Pediatr Phys Ther 19 (2007) 326-331.

[43] A. Muro-de-la-Herran, B. Garcia-Zapirain, A. Mendez-Zorrilla, Gait analysis methods: an overview of wearable and non-wearable systems, highlighting clinical applications, Sensors (Basel) 14(2) (2014) 3362-94. https://www.ncbi.nlm.nih.gov/pubmed/24556672.

[44] R. Baker, Gait analysis methods in rehabilitation, Journal of neuroengineering and rehabilitation 3 (2006) 4. https://www.ncbi.nlm.nih.gov/pubmed/16512912.

[45] B. Toro, C. Nester, P. Farren, A review of observational gait assessment in clinical practice, Physiother Theor Pract 19 (2003) 123-149.

[46] C.M. Kawamura, M.C. de Morais Filho, M.M. Barreto, S.K. de Paula Asa, Y. Juliano, N.F. Novo, Comparison between visual and three-dimensional gait analysis in patients with spastic diplegic cerebral palsy, Gait Posture 25(1) (2007) 18-24. https://www.ncbi.nlm.nih.gov/pubmed/16431106.

[47] C. Rathinam, A. Bateman, J. Peirson, J. Skinner, Observational gait assessment tools in paediatrics--a systematic review, Gait Posture 40(2) (2014) 279-85. https://www.ncbi.nlm.nih.gov/pubmed/24798609.

[48] A.M. Ong, S.J. Hillman, J.E. Robb, Reliability and validity of the Edinburgh Visual Gait Score for cerebral palsy when used by inexperienced observers, Gait Posture 28(2) (2008) 323-326. PM:18328710.

[49] S. Grunt, P.J. Van Kampen, M.M. van der Krogt, M.A. Brehm, C.A.M. Doorenbosch, J.G. Becher, Reproducibility of video screen measurements of sagittal joint angles during gait in patients with spastic cerebral palsy, JEGM 2010.

[50] T.A. Wren, G.E. Gorton, 3rd, S. Ounpuu, C.A. Tucker, Efficacy of clinical gait analysis: A systematic review, Gait Posture 34(2) (2011) 149-53. https://www.ncbi.nlm.nih.gov/pubmed/21646022.

[51] B. Ceulemans, Overall management of patients with Dravet syndrome, Dev Med Child Neurol 53 Suppl 2 (2011) 19-23. https://www.ncbi.nlm.nih.gov/pubmed/21504428.

[52] J.M. Rodda, I.E. Scheffer, J.M. McMahon, S.F. Berkovic, H.K. Graham, Progressive gait deterioration in adolescents with Dravet syndrome, Arch Neurol 69(7) (2012) 873-8. https://www.ncbi.nlm.nih.gov/pubmed/22409937. 
[53] J.J. Rilstone, F.M. Coelho, B.A. Minassian, D.M. Andrade, Dravet syndrome: seizure control and gait in adults with different SCN1A mutations, Epilepsia 53(8) (2012) 1421-8. https://www.ncbi.nlm.nih.gov/pubmed/22780858.

[54] A. Fasano, F. Borlot, A.E. Lang, D.M. Andrade, Antecollis and levodopa-responsive parkinsonism are late features of Dravet syndrome, Neurology 82(24) (2014) 2250-1.

[55] P. Kedem, D.M. Scher, Evaluation and management of crouch gait, Curr Opin Pediatr 28(1) (2016) 55-9. https://www.ncbi.nlm.nih.gov/pubmed/26709688. 


\begin{tabular}{|l|l|}
\hline User Query & (intellectual disability) AND gait \\
\hline Filter & Publication date: From 1/08/2013 \\
\hline Query Translation (Pubmed) & $\begin{array}{l}\text { ("intellectual disability"[MeSH Terms] OR ("intellectual"[All Fields] AND "disability"[All } \\
\text { Fields]) OR "intellectual disability"[All Fields]) AND ("gait"[MeSH Terms] OR "gait"[All } \\
\text { Fields]) AND ("2013/08/01"[PDAT] : "3000/12/31"[PDAT]) }\end{array}$ \\
\hline
\end{tabular}

Table 1: Search Details 


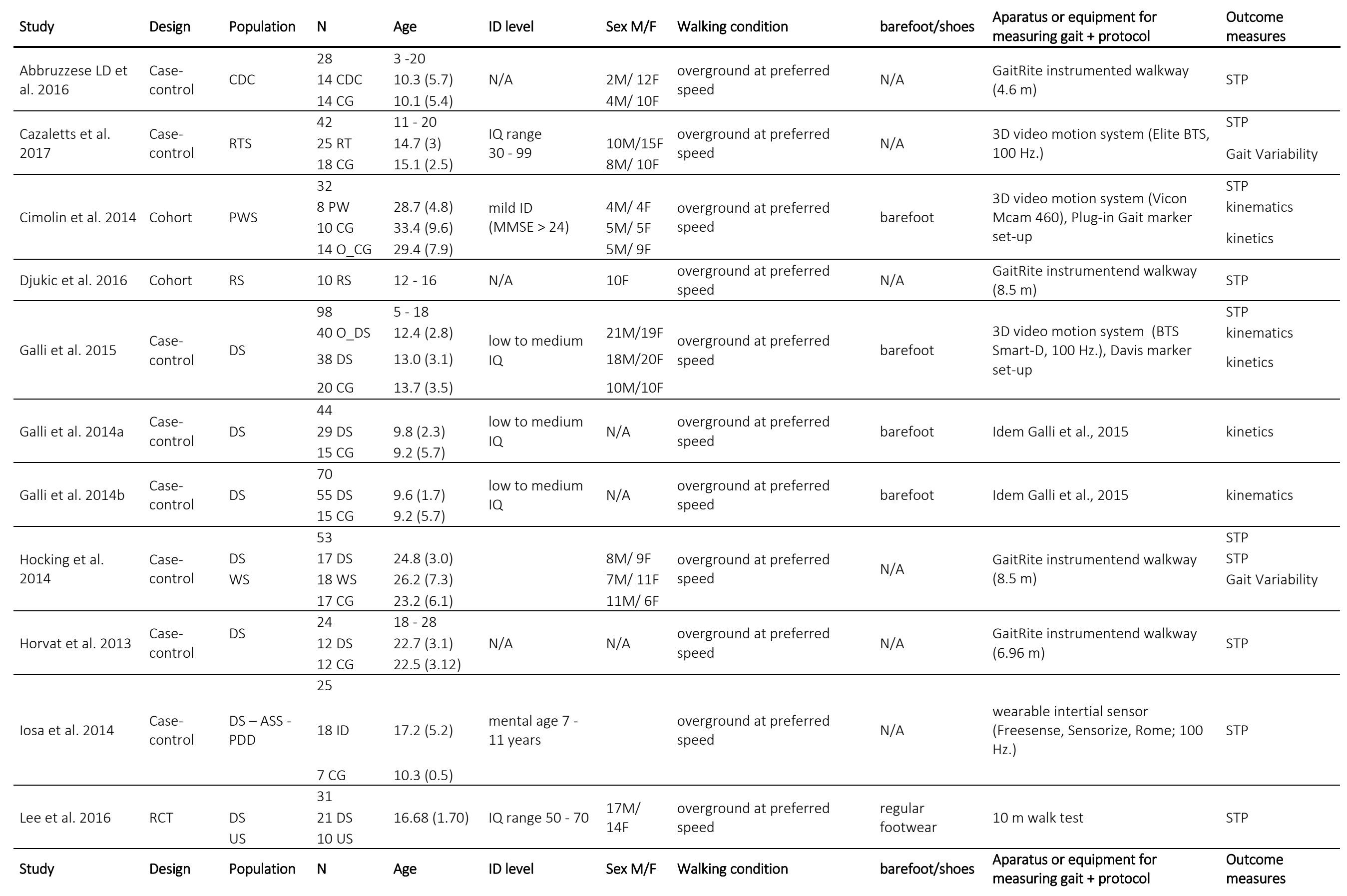




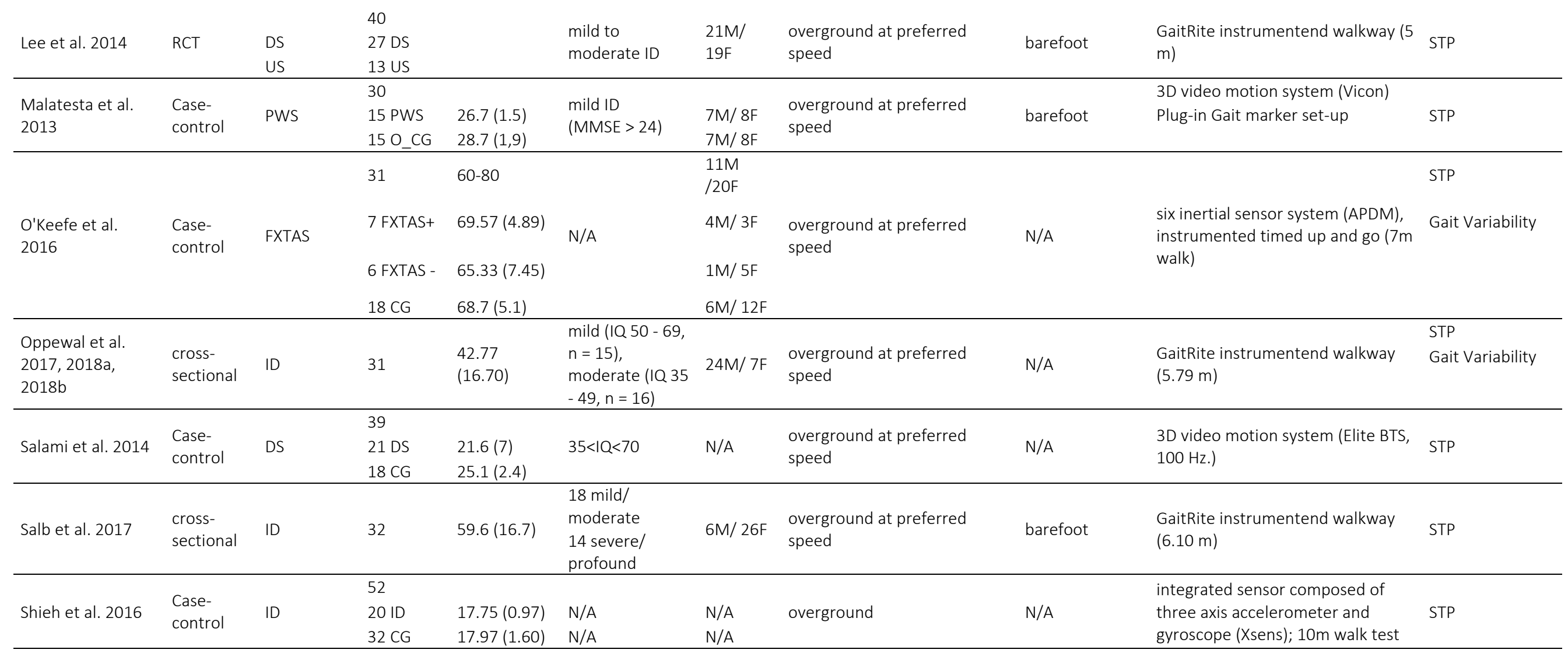

Table 1: Characteristics of studies describing overground and unperturbed locomotion

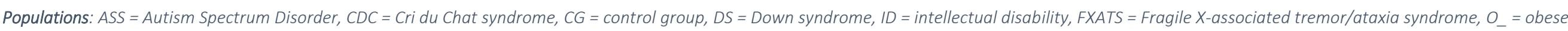
$P D D=$ Pervasive Developmental Disorder, PWS = Prader-Willi syndrome, $R S=$ Rett syndrome, $R T S=$ Rubinstein - Taybi syndrome, US = unknown syndrome, WS = Williams syndrome

Abbreviations: $N=$ sample size, $N / A=$ not available, $M / F=$ male/female, $S T P=$ spatio-temporal parameters 


\begin{tabular}{|c|c|c|}
\hline & VIDEO GAIT ANALYSIS & INSTRUMENTED GAIT ANALYSIS \\
\hline General & \multicolumn{2}{|c|}{$\begin{array}{l}\text { Recording of static posture and dynamic walking } \\
\text { On a sufficiently long walkway }(8-10 \mathrm{~m}) \text { at self-selected speed } \\
\text { In three anatomical planes } \\
\text { Barefoot and/or with shoes, orthotic devices or walking aids } \\
\text { Combined with structured physical examination }\end{array}$} \\
\hline Strengths & $\begin{array}{l}\text { Low budget } \\
\text { User friendly } \\
\text { Qualitative description } \\
\text { Estimate of kinematics }\end{array}$ & $\begin{array}{l}\text { Accurate } \\
\text { Reliable } \\
\text { Repeatable } \\
\text { Quantitative data }\end{array}$ \\
\hline Weaknesses & $\begin{array}{l}\text { Projection errors } \\
\text { Dependent on experience of the observer } \\
\text { Deprived visibility in the transverse plane } \\
\text { Less consistent than instrumented gait analysis }\end{array}$ & $\begin{array}{l}\text { Longer session duration } \\
\text { Good patient cooperation necessary } \\
\text { Discomfort for patient } \\
\text { Expensive equipment } \\
\text { High level of expertise of assessors needed } \\
\text { Specific knowledge for data interpretation required }\end{array}$ \\
\hline Indications & $\begin{array}{l}\text { Very young children, less cooperative children due to } \\
\text { deprived cognitive abilities or behavioural problems, } \\
\text { frequent monitoring in between IGA sessions }\end{array}$ & $\begin{array}{l}\text { Planning and follow up of surgery and orthotic } \\
\text { devices }\end{array}$ \\
\hline
\end{tabular}

Table 3: Overview of the different methods of gait analysis with their strengths, weaknesses and possible indications 
Figure 1: Flow diagram of the study selection process

Figure 2: Kinematic analysis of one patient at age $4 \mathrm{y} 4 \mathrm{~m}$ (solid lines) and $6 y 0 \mathrm{~m}$ (dashed lines). Mean joint angular time profiles, normalized to $100 \%$ of the gait cycle; red = left, blue = right; grey shaded area represents normative joint angular time profiles of typically developing children.

Figure 3: Kinetic analysis of one patient at age $4 \mathrm{y} 4 \mathrm{~m}$ (solid lines) and $6 \mathrm{y} 0 \mathrm{~m}$ (dashed lines). Mean joint angular time profile, net joint moment and net joint power of the ankle joint in the sagittal plane, normalized to $100 \%$ of the gait cycle; red = left, blue = right; grey shaded area represents normative joint angular time profiles of typically developing children. 
Figure 1 Flow diagram of the study selection process

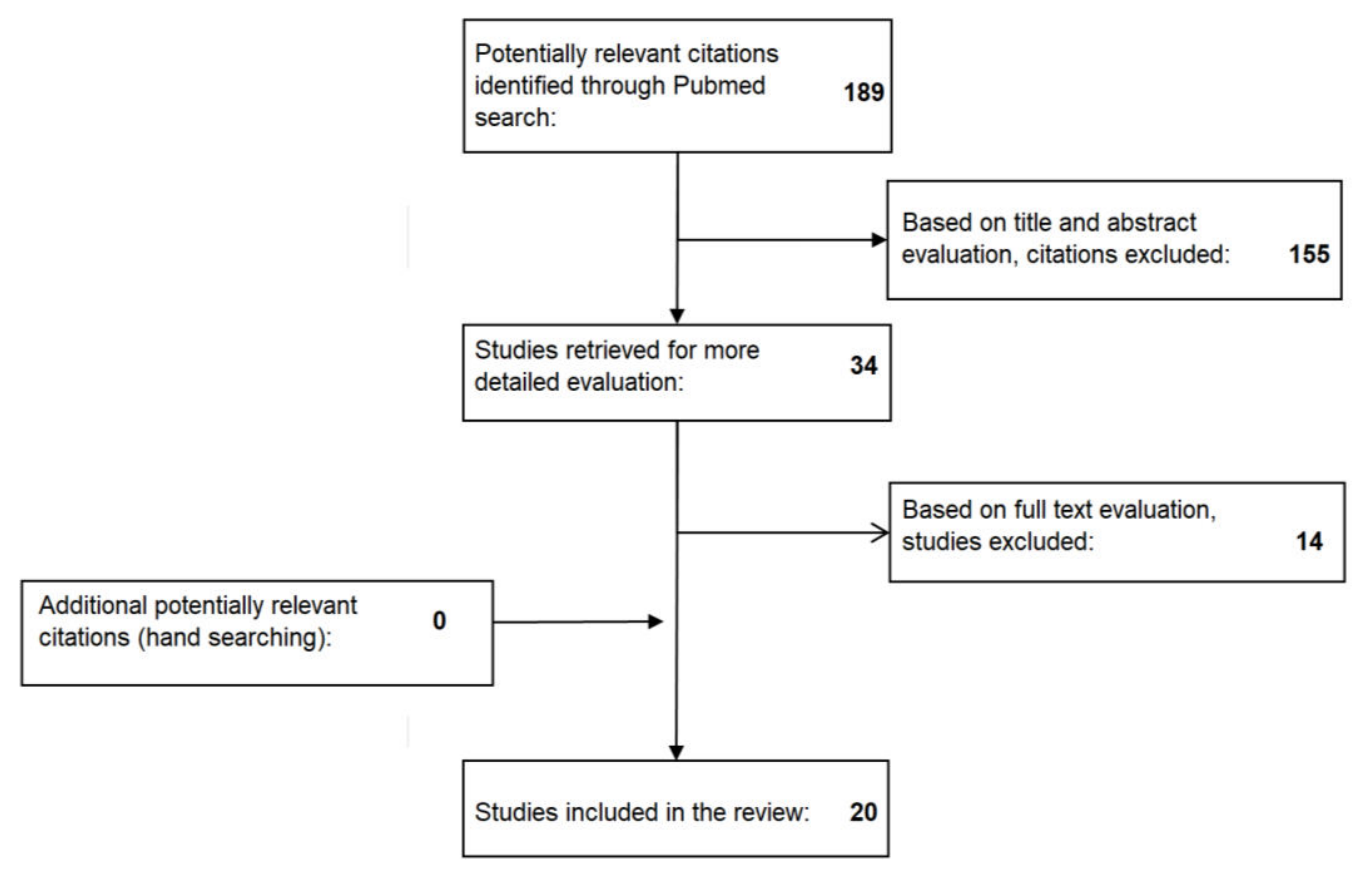




\section{Appendix 1: Gait characteristics in intellectual disabilities (ID) during unperturbed overground walking}

Table 1: Spatio-temporal parameters: mean (standard deviation). Underlined values in red differ significantly from CG. CG = control group, CDC $=$ Cri Du Chat syndrome, PWS = Prader Willi syndrome, $R S=$ Rett syndrome, DS = Down syndrome, WS = Williams syndrome, ID = intellectual disability of different causes, FXATS = Fragile X associated Ataxia and Tremor

\begin{tabular}{|c|c|c|c|c|c|c|c|c|c|c|c|c|}
\hline Authors & & $\begin{array}{l}\text { gait speed } \\
(\mathrm{m} / \mathrm{s})\end{array}$ & step time (s) & $\begin{array}{l}\text { cadence } \\
\text { (steps/min) }\end{array}$ & $\begin{array}{l}\text { step } \\
\text { length } \\
(\mathrm{cm}) \\
\end{array}$ & $\begin{array}{l}\text { normalised } \\
\text { step length }\end{array}$ & $\begin{array}{l}\text { stride } \\
\text { length } \\
(\mathrm{cm})\end{array}$ & $\begin{array}{l}\text { step width } \\
\text { (cm) }\end{array}$ & $\begin{array}{l}\text { stance } \\
(\%)\end{array}$ & $\begin{array}{l}\text { swing } \\
\text { (\%) }\end{array}$ & $\begin{array}{l}\text { double } \\
\text { support (s) }\end{array}$ & $\begin{array}{l}\text { double } \\
\text { support (\%) }\end{array}$ \\
\hline \multirow{2}{*}{$\begin{array}{l}\text { Abbruzzese LD et al. } \\
2016\end{array}$} & $C D C$ & $0.88(0.30)$ & & $\underline{135(30)}$ & $38.5(8.3)$ & & & $\underline{11.2(5.8)}$ & & & & $29.5(9.0)$ \\
\hline & CG & $1.02(0.12)$ & & $113(18)$ & $54.7(10.4)$ & & & $8.5(3.3)$ & & & & $26.5(3.5)$ \\
\hline \multirow[t]{2}{*}{ Cazaletts et al. 2017} & RTS & & & $123(16)$ & & $0.4(0.05)$ & & & & & & \\
\hline & CG & & & $115(8)$ & & $0.5(0.03)$ & & & & & & \\
\hline \multirow[t]{3}{*}{ Cimolin et al. 2014} & PWS & $0.88(0.09)$ & & $106(5)$ & & $0.48(0.04)$ & & & $60.7(1.6)$ & & & \\
\hline & OCG & $1.03(0.07)$ & & $116(5)$ & & $0.76(0.05)$ & & & $62.2(1.3)$ & & & \\
\hline & CG & $1.20(0.17)$ & & $117(5)$ & & $0.80(0.04)$ & & & $59.6(1.5)$ & & & \\
\hline Djukic et al. 2016 & RS & $\underline{0.627}$ & & & & & & & & & & \\
\hline \multirow[t]{3}{*}{ Galli et al. 2015} & $\begin{array}{l}\text { DS not } \\
\text { obese }\end{array}$ & $\underline{0.71(0.32)}$ & & $97(23)$ & & $\underline{0.33(0.08)}$ & & & $59(5.4)$ & & & \\
\hline & DS obese & $\underline{0.73(0.23)}$ & & $\underline{96(15)}$ & & $\underline{0.31(0.08)}$ & & & $\underline{63.7(3.5)}$ & & & \\
\hline & CG & $1.1(0.21)$ & & $115(5)$ & & $0.88(0.21)$ & & & $59.5(1.5)$ & & & \\
\hline \multirow[t]{3}{*}{ Hocking et al. 2014} & DS & $1.05(0.26)$ & $0.58(0.08)$ & 103 & $59.1(4.2)$ & & & 12.2 & & & $0.33(0.08)$ & \\
\hline & WS & $1.12(0.18)$ & $0.54(0.05)$ & 111 & $59.5(2.3)$ & & & 11.9 & & & $\underline{0.30(0.06)}$ & \\
\hline & CG & $1.3(0.14)$ & $0.55(0.05)$ & 109 & $70.9(2.7)$ & & & 8.6 & & & $0.27(0.06)$ & \\
\hline \multirow[t]{2}{*}{ Horvat et al. 2013} & DS & $1.30(0.10)$ & $0.52(0.02)$ & 115 & $67.3(6.4)$ & & & $\underline{9.0}$ & $64(0.02)$ & & 0.24 & \\
\hline & CG & $0.95(0.24)$ & $0.56(0.09)$ & 107 & $53.1(10.1)$ & & & 13.6 & $71(0.13)$ & & 0.31 & \\
\hline \multirow[t]{2}{*}{ losa et al. 2014} & ID & $1.20(0.28)$ & & & & & & & & & & \\
\hline & CG & $1.37(0.26)$ & & & & & & & & & & \\
\hline \multirow[t]{2}{*}{ Lee et al. 2016} & ID1 & $0.99(0.33)$ & & & & & & & & & & \\
\hline & ID2 & $0.94(0.34)$ & & & & & & & & & & \\
\hline \multirow[t]{2}{*}{ Lee et al. 2014} & ID1 & $0.91(0.19)$ & $0.57(0.04)$ & $135(8)$ & $50(9)$ & & & & & & & \\
\hline & ID2 & $0.89(0.22)$ & $0.55(0.44)$ & $135(9)$ & $49(12)$ & & & & & & & \\
\hline \multirow[t]{2}{*}{ Malatesta et al. 2013} & PWS & $0.98(0.03)$ & $0.53(0.01)$ & $115(2.4)$ & $\underline{51(1)}$ & & & & & & & 26 \\
\hline & CG & $1.20(0.02)$ & $0.53(0.01)$ & $115(1.8)$ & $63(1)$ & & & & & & & 24 \\
\hline \multirow[t]{3}{*}{ O'Keefe et al. 2016} & FXATS + & $1.09(0.18)$ & & $99(12)$ & & & & & & $37.5(2.2)$ & & $24.9(4.4)$ \\
\hline & FXATS - & $1.31(0.20)$ & & $114(9)$ & & & & & & $38.6(1.8)$ & & $22.8(3.8)$ \\
\hline & $\mathrm{CG}$ & $1.36(0.16)$ & & $116(9)$ & & & & & & $40.7(1.8)$ & & $18.5(3.6)$ \\
\hline Oppewal et al. 2017. & ID & $1.18(0.23)$ & $0.56(0.05)$ & $108(23)$ & $65.2(10.1)$ & & & $11.9(3.5)$ & $58.9(2.0)$ & $41.0(2.0)$ & & $18.1(4.1)$ \\
\hline
\end{tabular}


2018a. 2018b

Salami et al. 2014

DS

Salb et al. 2017

$\underline{0.66}$

Shieh et al. 2017

1.20

$\underline{42.2}$

58.6

19.9

$0.86(0.29)$

$1.14(0.47)$

$101(14)$

15.2

$102(27.9)$

120 
Table 2: Gait Variability as intra-subject coefficient of variation (\%). Underlined values in red differ significantly from CG. * COV were calculated from individual mean and standard deviation. CG = control group, RTS = Rubinstein - Taybi syndrome, DS = Down syndrome, WS = Williams syndrome, FXATS = Fragile X associated Ataxia and Tremor, ID = intellectual disability of various causes

\begin{tabular}{|c|c|c|c|c|c|c|c|c|}
\hline Authors & & COV step length & COV stride length & COV cadence & COV step width & COV stride time & COV step time & COV speed \\
\hline \multirow[t]{2}{*}{ Cazalets et al. 2017} & RTS & 8,8 & $\underline{7,7}$ & $\underline{5,8}$ & $\underline{18,9}$ & 6,3 & & \\
\hline & CG & $\overline{2,8}$ & 2 & $\overline{1,8}$ & 6,7 & $\overline{1,8}$ & & \\
\hline \multirow[t]{3}{*}{ Hocking et al. 2014} & DS & & & & & & 6,99 & 8,88 \\
\hline & WS & & & & & & 4,73 & 6,78 \\
\hline & CG & & & & & & 3,47 & 4,15 \\
\hline \multirow[t]{3}{*}{ O'Keefe et al. 2016} & FXATS + & & 3,99 & 4,48 & & & & 6,71 \\
\hline & FXATS - & & $\overline{2,07}$ & $\overline{2,36}$ & & & & $\overline{3,23}$ \\
\hline & CG & & 2,25 & 2,36 & & & & 3,08 \\
\hline Oppewal et al. 2018 & ID & $4,58^{*}$ & $4,04 *$ & & $21,13^{*}$ & $3,57^{*}$ & $3,57^{*}$ & $5,99^{*}$ \\
\hline
\end{tabular}


Table 3: Kinematic parameters: mean (standard deviation). Underlined values in red differ significantly from CG. CG = control group, PWS = Prader Willi syndrome, DS = Down syndrome; ROM = range of motion, $I C=$ initial contact, $\min =$ minimum, $\max =$ maximum, $S t=$ stance, $S W=$ swing, FPA = Foot Progression Angle

\begin{tabular}{|c|c|c|c|c|c|c|c|c|c|c|}
\hline \multirow{2}{*}{ Pelvis } & & \multicolumn{5}{|c|}{ Sagittal } & \multirow{2}{*}{\begin{tabular}{r|} 
Frontal \\
$\mathrm{ROM}^{\circ}$
\end{tabular}} & \multicolumn{3}{|c|}{ Transverse } \\
\hline & & & & & & $\mathrm{ROM}^{\circ}$ & & $\mathrm{ROM}^{\circ}$ & & \\
\hline \multirow[t]{3}{*}{ Cimolin et al. 2014} & PWS & & & & & $7.12(2.93)$ & & & & \\
\hline & CG obese & & & & & $5.67(3.46)$ & & & & \\
\hline & CG & & & & & $1.61(3.67)$ & & & & \\
\hline \multirow[t]{3}{*}{ Galli et al. 2015} & DS nonobese & & & & & $4.69(3.07)$ & $7.89(5.05)$ & $13.08(10.54)$ & & \\
\hline & DS obese & & & & & $4.65(1.91)$ & $7.34(6.25)$ & $12.86(10.66)$ & & \\
\hline & CG & & & & & $1.61(3.67)$ & $6.01(2.57)$ & $10.72(5.32)$ & & \\
\hline \multicolumn{2}{|l|}{ Hip } & $I C^{\circ}$ & & $\operatorname{minSt} t^{\circ}$ & & $\mathrm{ROM}^{\circ}$ & $\mathrm{ROM}^{\circ}$ & & & \\
\hline \multirow[t]{3}{*}{ Cimolin et al. 2014} & PWS & 49.24 (15.94) & & $8.64(10.78)$ & & $39.25(6.86)$ & & & & \\
\hline & OCG & $43.50(11.23)$ & & $-3.46(10.71)$ & & $43.45(4.10)$ & & & & \\
\hline & CG & $27.22(7.54)$ & & $-11.92(7.68)$ & & $45.92(5.36)$ & & & & \\
\hline \multirow[t]{3}{*}{ Galli et al. 2015} & DS nonobese & $36.30(11.98)$ & & $\underline{0.38(10.92)}$ & & $37.16(8.58)$ & $12.94(6.39)$ & & & \\
\hline & DS obese & $36.58(14.44)$ & & $2.29(8.98)$ & & $36.67(9.55)$ & $13.67(6.22)$ & & & \\
\hline & CG & $27.22(7.54)$ & & $-13.92(7.68)$ & & $44.92(5.36)$ & $11.92(6.13)$ & & & \\
\hline \multicolumn{2}{|l|}{ Knee } & $\mathrm{IC}^{\circ}$ & $\operatorname{minSt}{ }^{\circ}$ & & $\operatorname{maxSw^{\circ }}$ & $\mathrm{ROM}^{\circ}$ & & & & \\
\hline \multirow[t]{3}{*}{ Cimolin et al. 2014} & PWS & $10.99(6.29)$ & $-0.49(4.19)$ & & $55.84(7.04)$ & $56.75(6.33)$ & & & & \\
\hline & CG obese & $4.93(7.25)$ & $-2.2(5.94)$ & & $54.84(7.34)$ & $58.23(4.42)$ & & & & \\
\hline & CG & $4.06(6.63)$ & $0.12(3.82)$ & & $59.01(6.18)$ & $60.28(6.31)$ & & & & \\
\hline \multirow[t]{3}{*}{ Galli et al. 2015} & DS nonobese & $7.11(11.68)$ & 6.29 (11.49) & & $52.52(8.31)$ & $49.08(9.57)$ & & & & \\
\hline & DS obese & $5.94(12.21)$ & $5.21(9.06)$ & & $51.34(7.01)$ & $44.63(10.62)$ & & & & \\
\hline & CG & $4.06(6.63)$ & $0.12(3.82)$ & & $59.01(6.18)$ & $60.28(6.31)$ & & & & \\
\hline \multicolumn{2}{|c|}{ Ankle \& Foot Progression Angle } & $\mathrm{IC}^{\circ}$ & $\operatorname{maxSt^{\circ }}$ & $\operatorname{minSt}{ }^{\circ}$ & $\operatorname{maxSw^{\circ }}$ & $\mathrm{ROM}^{\circ}$ & & Mean $\mathrm{FPA}^{\circ}$ & $\mathrm{FPA} \mathrm{St}^{\circ}$ & FPA Sw $^{\circ}$ \\
\hline \multirow[t]{3}{*}{ Cimolin et al. 2014} & PWS & $-1.61(9.89)$ & $13.59(6.77)$ & $-7.18(11.83)$ & $11.53(9.61)$ & $20.78(5.04)$ & & & & \\
\hline & CG obese & $-1.45(7.26)$ & $13.95(3.34)$ & $-15.85(6.61)$ & $5.08(2.36)$ & $29.81(6.88)$ & & & & \\
\hline & CG & $1.81(4.87)$ & $12.91(5.97)$ & $-18.98(6.19)$ & $8.63(9.93)$ & $27.72(6.56)$ & & & & \\
\hline \multirow[t]{3}{*}{ Galli et al. 2015} & DS nonobese & $-2.97(5.81)$ & $13.37(9.05)$ & $-4.74(7.47)$ & $8.91(8.08)$ & $17.56(8.40)$ & & $-20.69(11.71)$ & & \\
\hline & DS obese & $-3.29(6.60)$ & $10.85(7.16)$ & $-4.59(8.55)$ & $\underline{4.65(8.55)}$ & $15.99(7.21)$ & & $-22.17(15.19)$ & & \\
\hline & CG & $1.81(4.87)$ & $19.91(5.97)$ & $-8.98(6.19)$ & $8.63(9.93)$ & $27.72(6.56)$ & & $-14.88(8.35)$ & & \\
\hline \multirow{3}{*}{ Galli et al. 2014b } & DS normal/high arch & & & & & & & & $-15.05(9.99)$ & $-12.52(8.02)$ \\
\hline & DS low arch & & & & & & & & $-20.73(10.48)$ & $-21.85(11.53)$ \\
\hline & CG & & & & & & & & $-13.51(3.64)$ & $-16.12(4.23)$ \\
\hline
\end{tabular}


Table 4: Kinetic parameters: mean (standard deviation). Underlined values in red differ significantly from CG. CG = control group, PWS = Prader Willi syndrome, DS = Down syndrome

\begin{tabular}{|c|c|c|c|c|}
\hline Ankle & & $\begin{array}{l}\max \text { PF Moment } \\
(\mathrm{Nm} / \mathrm{kg})\end{array}$ & $\begin{array}{l}\text { Max Power } \\
\text { Generation (W/kg) }\end{array}$ & $\begin{array}{l}\text { Max normalized } \\
\text { Power Generation }\left(W^{*} s / k g . m\right)\end{array}$ \\
\hline \multirow[t]{3}{*}{ Cimolin et al. 2014} & PWS & $1.02(0.17)$ & $1.59(0.51)$ & $1.82(0.55)$ \\
\hline & CG & $1.47(0.13)$ & $3.01(0.52)$ & $2.95(0.80)$ \\
\hline & CG obese & $1.68(0.25)$ & $3.73(0.71)$ & $3.10(1.06)$ \\
\hline \multirow[t]{3}{*}{ Galli et al. 2015} & DS nonobese & $1.08(0.38)$ & $1.59(0.96)$ & \\
\hline & DS obese & $0.98(0.35)$ & $1.48(0.78)$ & \\
\hline & CG & $1.49(0.25)$ & $3.73(0.71)$ & \\
\hline \multirow[t]{3}{*}{ Galli et al. 2014a } & DS high/normal arch & $0.99(0.17)$ & $1.60(0.65)$ & \\
\hline & DS low arch & $\underline{0.86(0.27)}$ & $1.20(0.52)$ & \\
\hline & CG & $1.29(0.23)$ & $3.06(1.16)$ & \\
\hline
\end{tabular}



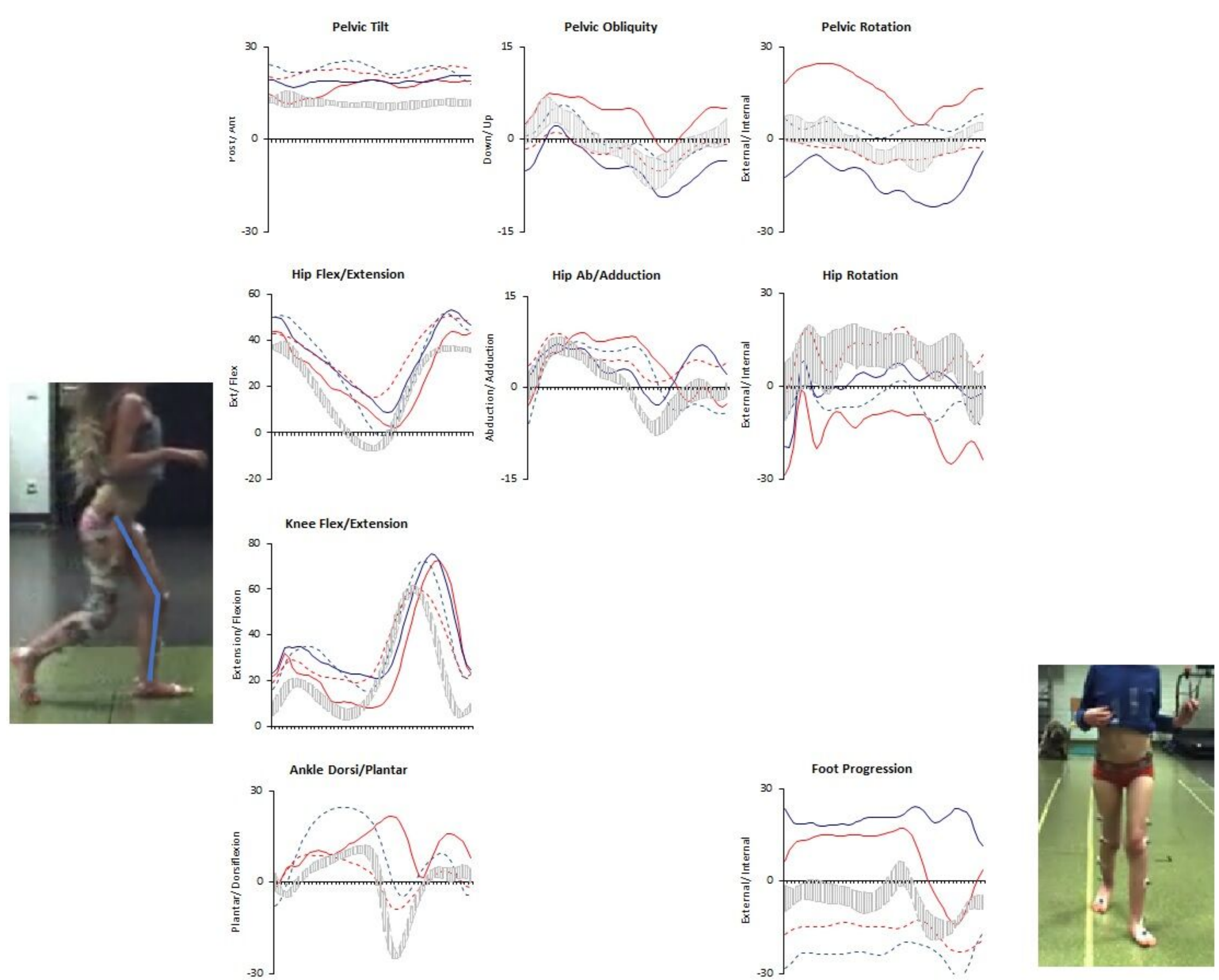

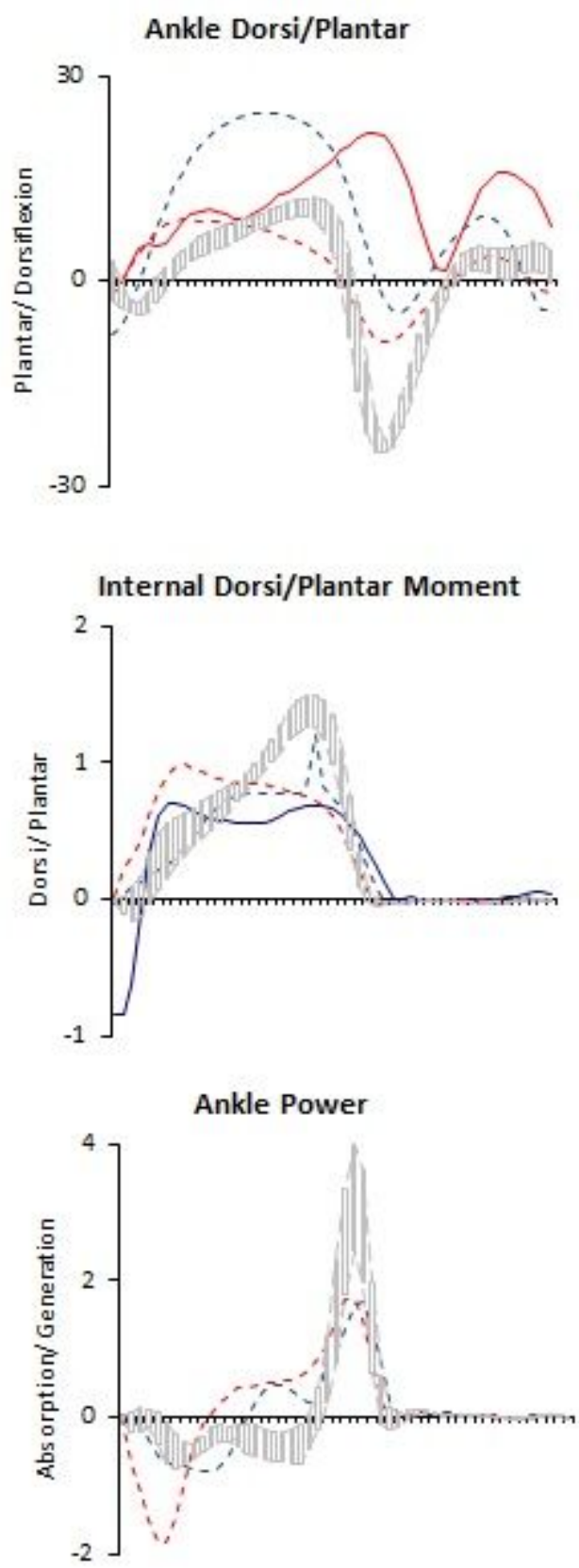


\section{Appendix 2: Gait characteristics in intellectual disabilities (ID) during dual tasking}

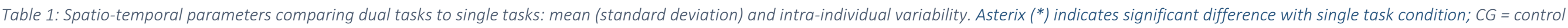
group, $C D C=$ Cri Du Chat syndrome, DS = Down syndrome, WS = Williams syndrome, ID = intellectual disability of different causes; $C O \mathrm{~V}=$ coefficient of variation, $S D=$ standard deviation

\begin{tabular}{|c|c|c|c|c|c|c|c|c|c|c|c|}
\hline & & & $\begin{array}{l}\text { gait speed } \\
(\mathrm{m} / \mathrm{s})\end{array}$ & Step time (s) & $\begin{array}{l}\text { cadence } \\
\text { (steps/min) }\end{array}$ & $\begin{array}{l}\text { step length } \\
\text { (cm) }\end{array}$ & $\begin{array}{l}\text { Step Width } \\
(\mathrm{cm})\end{array}$ & $\begin{array}{l}\text { double support } \\
\text { (s) }\end{array}$ & $\begin{array}{l}\text { COV } \\
\text { speed }\end{array}$ & $\begin{array}{l}\text { COV step } \\
\text { time }\end{array}$ & $\begin{array}{l}\text { SD step } \\
\text { length }\end{array}$ \\
\hline \multirow{6}{*}{$\begin{array}{l}\text { Abbruzzese et al. } \\
2016\end{array}$} & \multirow[t]{3}{*}{$\mathrm{CDC}$} & Single task & $0.88(0.30)$ & & $134.6(29.7)$ & $38.5(8.3)$ & $11.2(5.8)$ & $29.5(9.0) \%$ & & & \\
\hline & & Tray & $0.77(0.25)$ & & $127.1(23.4)^{*}$ & 37.1 (8.9) & $12.1(4.3)$ & $30.8(8.9) \%$ & & & \\
\hline & & Pitcher & $0.66(0.19)^{*}$ & & $119.9(23.9)^{*}$ & $33.3(8.2)^{*}$ & $10.9(5.6)$ & $35.4(6.4) \% *$ & & & \\
\hline & \multirow[t]{3}{*}{ CG } & Single task & $1.02(0.12)$ & & $112.8(17.7)$ & $54.7(10.4)$ & $8.5(3.3)$ & $26.4(3.5) \%$ & & & \\
\hline & & Tray & $0.79(0.19)^{*}$ & & $98.1(13.3)^{*}$ & $47.4(10.6)^{*}$ & $8.9(2.6)$ & $31.3(3.8) \% *$ & & & \\
\hline & & Pitcher & $0.84(0.24)^{*}$ & & $97.7(14.2)^{*}$ & $50.8(11.0)^{*}$ & $7.5(4.0)$ & $30.1(4.4) \% *$ & & & \\
\hline \multirow{12}{*}{$\begin{array}{l}\text { Hocking et al. } \\
2014\end{array}$} & \multirow[t]{4}{*}{ DS } & Single task & $1.05(0.26)$ & $0.58(0.08)$ & & $59.0(9.8)$ & $12.2(4.2)$ & $0.33(0.08)$ & $8.9(3.7)$ & $6.9(1.8)$ & \\
\hline & & Verbal fluency & $*$ & $*$ & & $*$ & ns & $*$ & * & $*$ & \\
\hline & & Digit span short & ns & ns & & ns & ns & ns & ns & ns & \\
\hline & & Digit span long & ns & ns & & ns & bs & ns & ns & ns & \\
\hline & \multirow[t]{4}{*}{ WS } & Single task & $1.12(0.18)$ & $0.54(0.05)$ & & 59.51 (7.09) & $11.95(2.30)$ & $0.30(0.06)$ & $6.8(2.5)$ & $4.7(1.2)$ & \\
\hline & & Verbal fluency & ns & ns & & ns & ns & ns & ns & ns & \\
\hline & & Digit span short & $*$ & ns & & ns & $*$ & ns & ns & & \\
\hline & & Digit span long & ns & ns & & ns & $*$ & ns & $*$ & ns & \\
\hline & \multirow[t]{4}{*}{$\mathrm{CG}$} & Single task & $1.30(13.85)$ & $0.55(0.05)$ & & $70.94(6.26)$ & $8.62(2.69)$ & $0.27(0.06)$ & 4.2 (1.9) & $3.5(1.7)$ & \\
\hline & & Verbal fluency & * & * & & $*$ & ns & $*$ & * & $*$ & \\
\hline & & Digit span short & $*$ & ns & & ns & $*$ & ns & ns & $*$ & \\
\hline & & Digit span long & ns & ns & & ns & $*$ & ns & $*$ & ns & \\
\hline \multirow{10}{*}{$\begin{array}{l}\text { Horvat et al. } \\
2013\end{array}$} & \multirow[t]{5}{*}{ DS } & Single task & $1.30(0.10)$ & $0.52(0.02)$ & & $67.3(6.4)$ & $9.0(1.4)$ & $0.24(0.02)$ & & & \\
\hline & & Plate \& Cup & $1.32(0.09)$ & $0.51(0.02)^{*}$ & & $67.8(5.9)^{*}$ & $9.2(1.5)^{*}$ & $0.23(0.01)^{*}$ & & & \\
\hline & & Tray \& Cup & $1.37(0.16)$ & $0.50(0.02)^{*}$ & & $68.9(7.7)$ & $9.2(1.9)^{*}$ & $0.22(0.01)^{*}$ & & & \\
\hline & & Phone & $1.24(0.14)^{*}$ & $0.53(0.02)$ & & $65.7(7.4)^{*}$ & $9.7(1.8)^{*}$ & $0.24(0.02)$ & & & \\
\hline & & Buttoning shirt & $1.23(0.11)^{*}$ & $0.52(0.02)$ & & $64.5(6.1)^{*}$ & $10.7(2.6)^{*}$ & $0.25(0.02)$ & & & \\
\hline & \multirow[t]{5}{*}{$\mathrm{CG}$} & Single task & $0.95(0.24)$ & $0.56(0.09)$ & & $53.1(10.1)$ & $13.6(5.3)$ & 0.31 & & & \\
\hline & & Plate \& Cup & $0.95(0.27)$ & $0.57(0.15)^{*}$ & & $51.6(11.7)$ & $15.0(5.9)^{*}$ & $0.35(0.23)^{*}$ & & & \\
\hline & & Tray \& Cup & $0.87(0.25)$ & $0.57(0.17)^{*}$ & & $46.6(7.6)$ & $14.8(5.4)^{*}$ & $0.36(0.26)^{*}$ & & & \\
\hline & & Phone & $0.77(0.21)$ & $0.61(0.15)$ & & $44.7(7.7)^{*}$ & $14.8(5.8)^{*}$ & $0.41(0.25)$ & & & \\
\hline & & Buttoning shirt & $0.77(0.26)$ & $0.64(0.30)$ & & $42.6(8.8)^{*}$ & $15.0(5.8)^{*}$ & $0.48(0.50)$ & & & \\
\hline $\begin{array}{l}\text { Oppewal et al. } \\
2017\end{array}$ & ID & $\begin{array}{l}\text { Single task } \\
\text { Talking }\end{array}$ & $\begin{array}{l}1.18(0.23) \\
*\end{array}$ & $\begin{array}{l}0.56(0.05) \\
*\end{array}$ & $\begin{array}{l}108(10) \\
*\end{array}$ & $\begin{array}{l}65.3(10.1) \\
*\end{array}$ & $\begin{array}{l}11.9(3.5) \\
\mathrm{ns}\end{array}$ & $\begin{array}{l}0.20(0.06) \\
*\end{array}$ & $\begin{array}{l}7.1(2.8) \\
\text { ns }\end{array}$ & $\begin{array}{l}0.02(0.01) \\
\mathrm{ns}\end{array}$ & $\begin{array}{l}2.99(0.98) \\
*\end{array}$ \\
\hline
\end{tabular}

